NASA CR-62004

DSR 9991-2

A SAMPLED-DATA PURSUIT TRACKING MODEL

By J. G. Kreifeldt

January 15, 1965

Distribution of this report is provided in the interest of information exchange. Responsibility for the contents resides in the author or organization that prepared it.

Prepared under Grant No. NsG 107-61 by MASSACHUSETTS INSTITUTE OF TECHNOLOGY Cambridge, Mass.

for

NATIONAL AERONAUTICS AND SPACE ADMINISTRATION 


\title{
A SAMPLED-DATA PURSUIT TRACKING MODEL
}

\author{
J. G. Kreifeldt
}

\section{Summary}

A sampled-data pursuit hand tracking model for the human operator is developed and tested. The model embodies the simplest a priori assumptions about human tracking behavior. The analytical model is presented along with the experimentally determined frequency transfer characteristics of an analog computer built to have the same transmittance as the mathematical model. Generally good agreement was obtained in matching the model's frequency and time domain responses to those of a well-trained human tracking in pursuit fashion an input power spectrum flat to $0.64 \mathrm{cps}$. 
Introduction

Human tracking studies performed in laboratories have distinguished two experimental modes: compensatory tracking and pursuit tracking. The first mode presents the operator with information only of the instantaneous error between command signal (input) and system response (output). The second mode presents the operator with a direct representation of both the input and the output simultaneously.

Elkind's ${ }^{1}$ systematic work has demonstrated that the frequency response characteristics obtained from human pursuit and compensatory tracking of random or quasi-random inputs differ markedly. Figure 1 taken from this work shows the differences in amplitude and phase characteristics for these two modes. Several flat input spectra up to $0.64 \mathrm{cps}$ were used for both modes. These results were obtained with negligible control dynamics external to the human.

The human tracking in the compensatory mode is considered a singleinput, single-output operator acting on the error in standard, servo-mechanism fashion. In accordance with the realities of the situation the human pursuit tracker should be considered a two-input, single-output operator and the discrepancy in his behavior from compensatory tracking should be explained on this basis.

Compensatory tracking modeling has received by far the major share of attention to date. This may be attributed to

1. The compensatory model lends itself readily to analytic investigation. For instance, in the compensatory model the open-loop (or operator's) continuous linear transfer characteristics can be deduced from the closed loop system frequency response characteristics. This is not true for the pursuit model in which the human is a two-input, singleoutput operator.

2. The past work in continuous compensatory models has provided a background and an impetus for further compensatory modeling even though a recent trend is toward the inclusion of discrete or sampled-data 

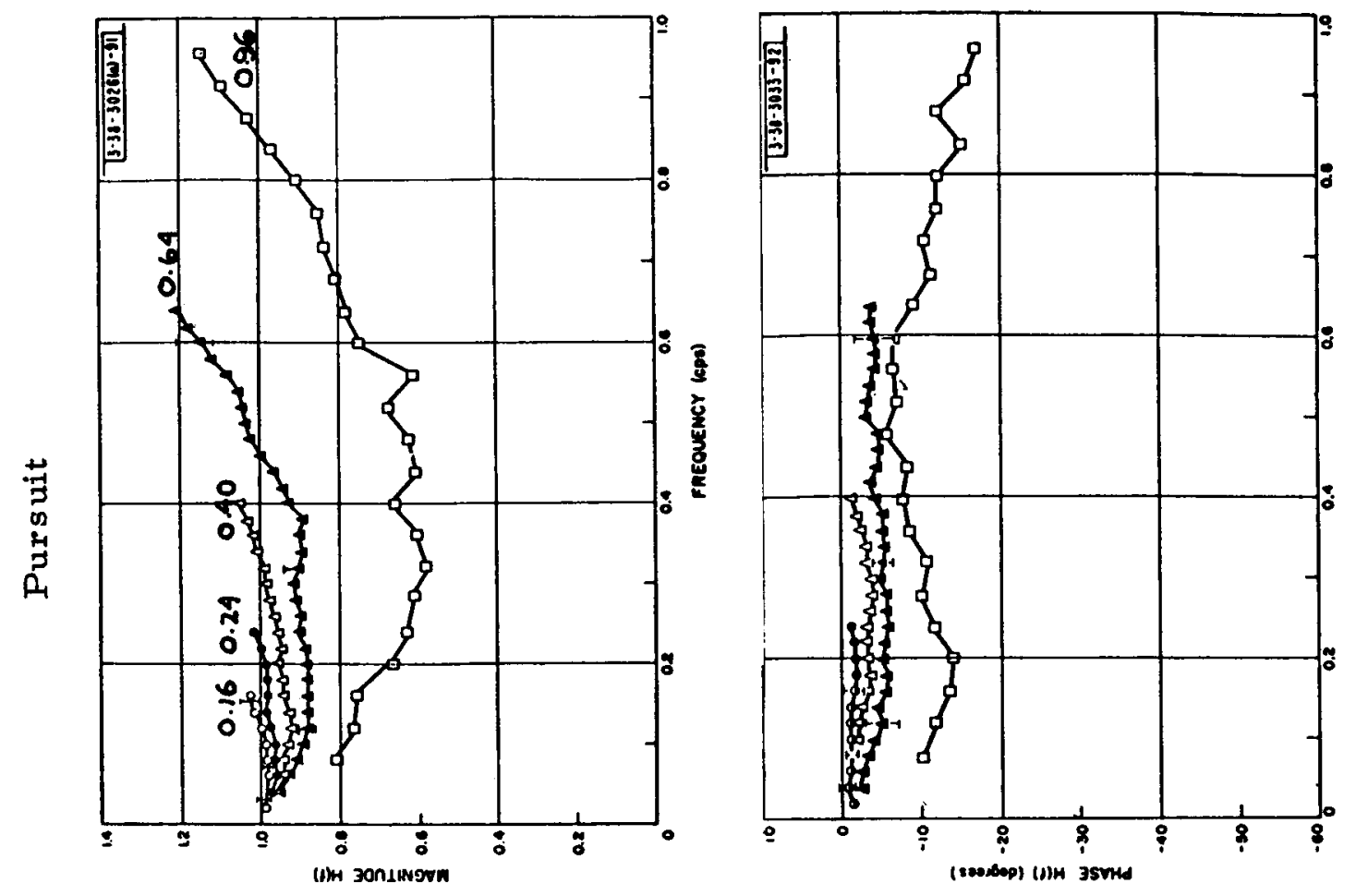

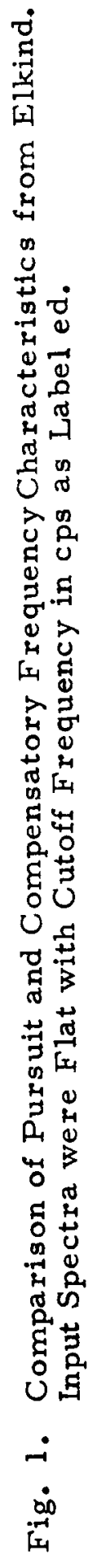
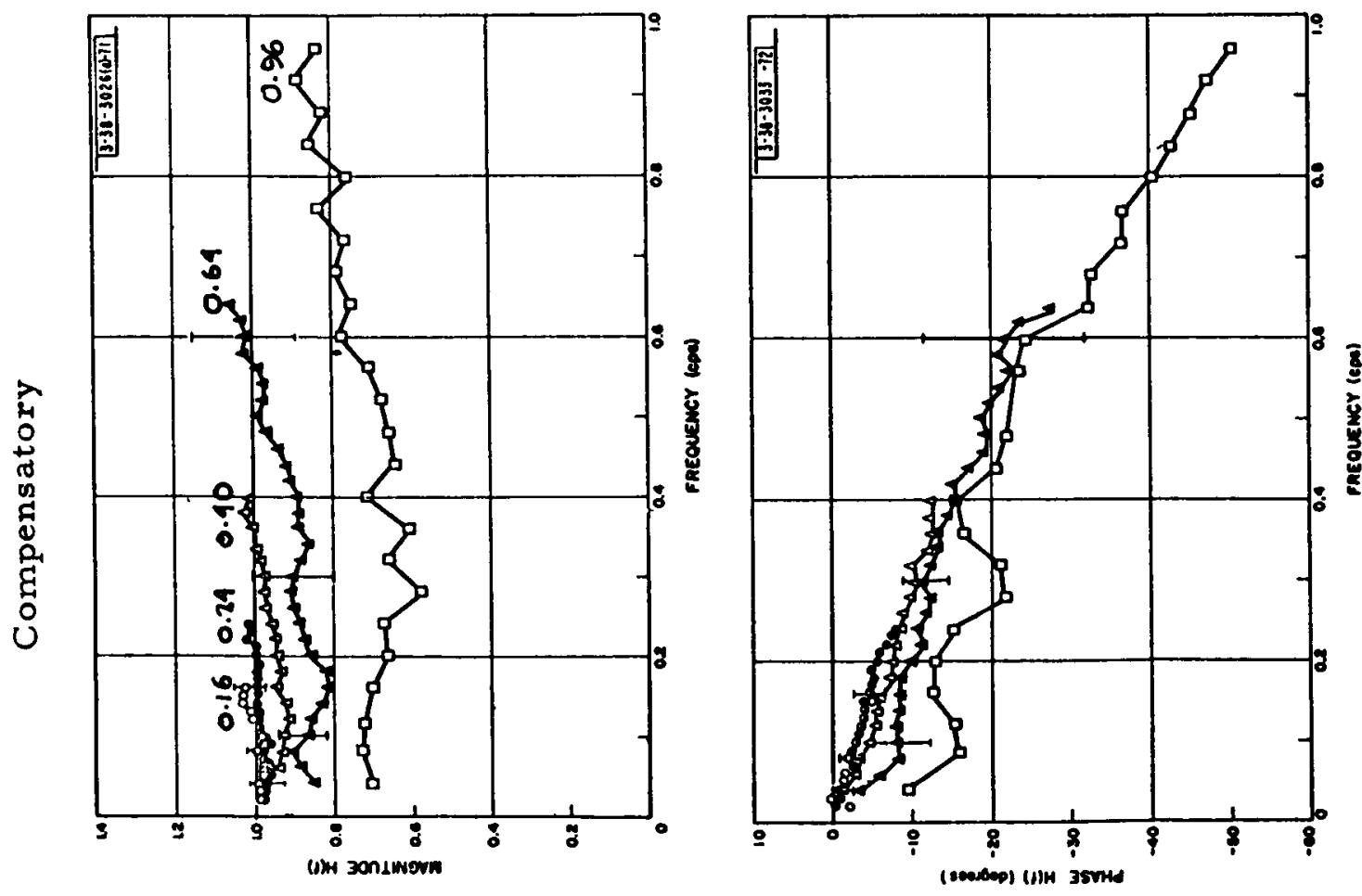
mechanis ins in the compensatory models. In such

model: the postulated sampled-data operators are not deducible analytically from closed loop syotem frequency characterietice.

3. It may be argued that compenvatory modeling furnishes all the interesting aspects of human tracking behavior, and, since it leads to a simpler model than pursutt tracking, it is best to stick to the compensatory case.

It is the contention of this paper that a pursult tracking model is worthy of study for the following reasons:

1. The marked difference in behavior between compensatory and pursuit tracking sould be explained.

2. No satisfactory pursuit model exists in the literature to date.

3. A pursult model would furnish an opportunity to extend application of human tracking over what is now underatood in terms of compenatory modele.

4. A sattsfactory pursuit model would provide a starting point for more sophieticated modeling of situations in which several inputs are presented to the human.

Previous Pursutt Model

The only pursuit model to appear in the literature has been the one developed by Elkind and ts shown in Fig. 2. The model has the form of a conditional servo. From examination of empirical records he hypothesized that $P_{1}(f)$ is a predictor of the form:

$$
P_{1}(f)=b_{0}+b_{1}(j 2 \pi f)
$$

which operated directly on the input to produce position and veloctty information. $G_{1}(f)$ he constructed as: 

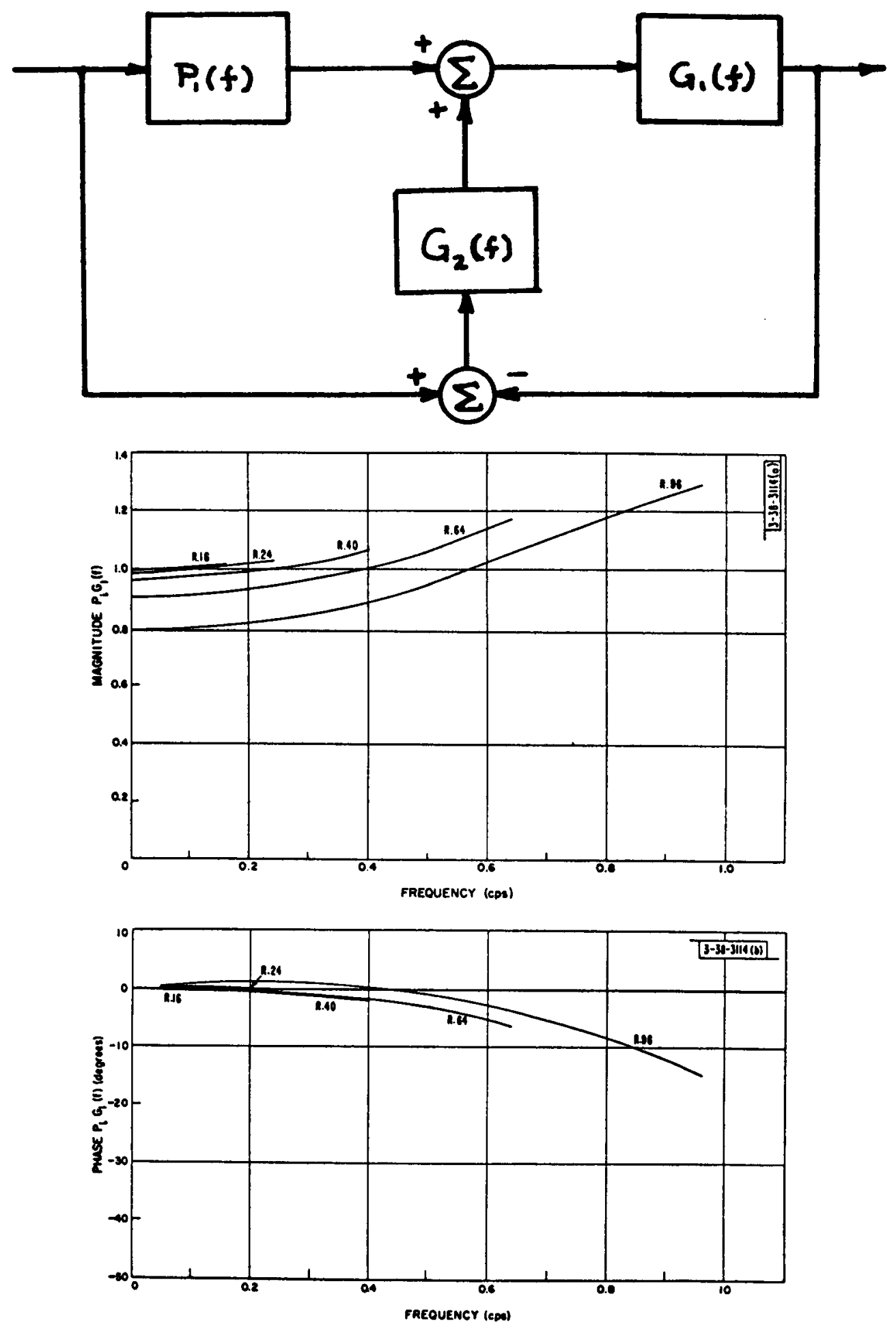

Fig. 2. Elkind's Pursuit Block Diagram Model and the Open-Loop Characteristics of $P_{1} G_{1}(f)$. 


$$
G_{1}(f)=\frac{e^{-j 2 \pi f(0.13)}}{\left(\frac{j f}{4}\right)^{2}+1.6\left(\frac{j f}{4}\right)+1}
$$

This is essentially the muscle response with the 0.13 second delay found for compensatory tracking. This second order filter will give nearly the same step response (except for the 0.13 second delay) as is observed in step tracking. The function $G_{2}(f)$ which is to operate on the error is

$$
G_{2}(f)=\frac{K}{\left(\frac{j f}{f_{1}}+1\right)\left(\frac{j f}{f_{2}}+1\right)}
$$

where the constants were never specified. The predictor $P_{1}(f)$ was necessary to offset the inherent pure delay of 0.13 second and the lag in the muscle response.

For analytical purposes Elkind set $G_{2}(f)$ equal to zero assuming that if $P_{1} G_{1}(f)$ was near unity the error operator would have little effect. Therefore, the model he tested consisted only of $P_{1}(f)$ cascaded with $G_{1}(f)$ where $G_{1}(f)$ was equivalent to a pure delay of 0.194 second. The derived frequency response of the model with these assumptions is pictured below the model in Fig. 2.

A recent paper by Elkind, Kelly and Payne ${ }^{2}$ develops another compensatory hand tracking model. In this model, following Young ${ }^{3}$ there is a path called the pursuit channel. However, this model is not a pursuit tracking model in the present sense since it operates only on the error.

Desirable Properties of a Pursuit Mode1

A model was sought which would fulfill the following requirements:

1. Frequency and Time Domain Fit to Data

The model should duplicate as closely as possible the frequency transfer characteristics for pursuit tracking shown in Fig. 1. Since it was not expected that an exact frequency match 
would be possible, the model should provide a close match to Interesting portions of the time tracings of human pursuit tracking response.

2. Linearity and Stationarity

The assumption of linearity seemed justified on the basis of previous experimental evidence as well as the basis of practical applicability afforded by a linear model. Although the human tracker obviously possesses great adaptability and recent attempts have been made to simulate some of these adaptive features, it was not considered feasible to attempt modeling his time-variant and adaptive characteristics.

3. Discrete or Intermittant Operations

The prior work of Bekey, ${ }^{4}$ Young $^{3}$ and Wescott and Lemay ${ }^{5}$ in compensatory modeling has proven the feasibllity and provided the justification for the inclusion of sampled-data (or intermittant) operators in human tracking models. The evtdence for these discrete operators rest. more strongly on examination of actual time tracings of human tracking responses rather than on frequency response characteristica. Some evidence for discrete operation in the human are given below.

Typically, a subject's response to a step input consists of a pure delay ranging from approximately 0.2 second to 0.35 second followed by a series of double parabola-like responses until the subject is satisfled with the remaining error. A complete double-parabola segment of the response is approximately 0.2 second in duration. Westcott and Lemay, who pointed out this parabola type response, add this as evidence for discrete programming in tracking continuous ignale.

Young similarly found very strong evidence for discrete operation in control of eye movements and succeeded to a high degree in modeling eye tracking movements through the use of discrete operators. 
Figure 3 show a egment of the velocity records a pursuit tracker's response to a contlnuous randominput. Apparent segmenting of the velocity record can be dis cerned with a frequency of about 5 segments per second. The regularity of these occurrences is taken as further evidence of the execution of preprogrammed movements discretely formulated.

\section{Conditional Servo System}

In the pursuit tracking mode it is a fair assumption that the tracker performs some operations on the input directly -ince it is available to him in uncontaminated form, and that he also compares the input and output to get some measure of the error. The total program to be executed by the muscle syatem is derived from summing the separate operations performed on the input and on the error. Tht. leads to the conditionsl servo syatem form as used by Elkind (Fig. 2), where the error nulling feedback is conditional upon there being an unaatiafactory tranofer between input and output through the direct forward loop.

\section{Element: of the Model}

To achieve the above properties a sampled-data puraut model wa: built as followe. (See Fig. 4):

1. There is an inherent pure delay in the human of 0.2 second as observed from transient tracking (as in step tracking). This is, of course, only approximate and if the model is to match a particular person, it would be better to we his particular delay time observed from his transient response records. The 0.2 second delay is the time from the beginning of formulation of the program to the beginning of it execution. In Fig. 4 this delay is sown to be effective in both an error sensitive loop and in input sensitive loop. 

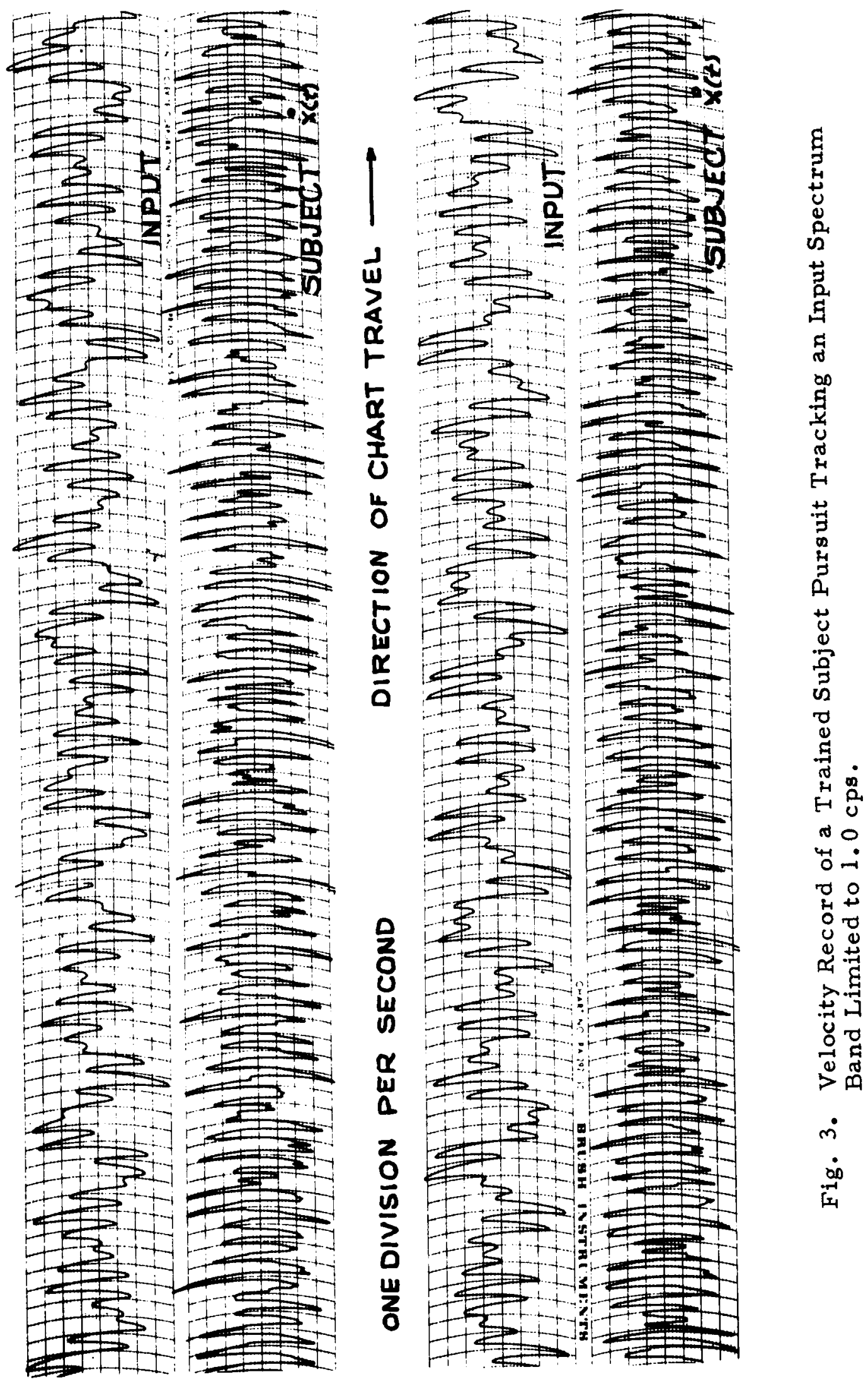


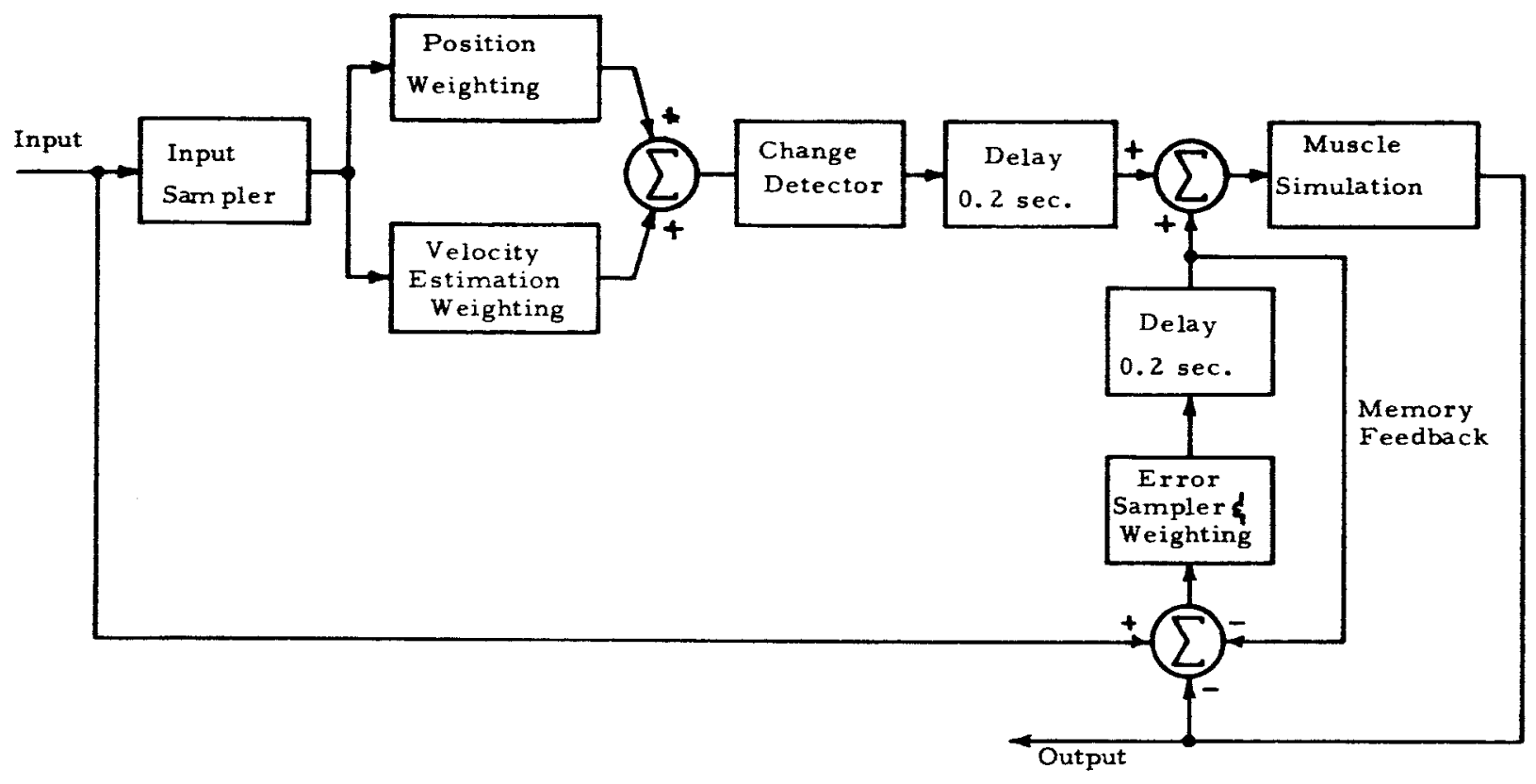

Fig. 4. Block Diagram of Sampled-Data Pursuit Tracking Model. 
2. The human tracks random continuous signals by preprogramming and executing discrete muscle movements. The discrete programs are 0.2 second in duration. The preprogrammed movements made from weighted input position and velocity estimation and error information are executed by a "muscle" which consists of a single nonresetting integrator, where the program segments fed to the "muscle" are flat-topped pulses of 0.2 second duration. The "muscle" therefore produces a ramp during the pulse holding at the final value until the next pulse (See Fig. 5).

3. To offset the pure 0.2 second delay time and the time required by the muscle for complete execution of a program, the operator attempts to predict the future position of the input by extracting and combining weighted estimates of the present input velocity and position. The operator can obtain an estimate of the instantaneous velocity by noting the difference between the present sample and the previous (stored) sample of the input, both samples taken in the forward path. This is actually an estimate of the average velocity during a sample period but for frequencies which are low compared to the sampling frequency, this can be a good instantaneous velocity estimate. ${ }^{+}$

\footnotetext{
+ Westcott and Lemay and Elkind et al incorporated into their compensatory hand tracking models a double-integrator "muscle" driven by a force program composed of a flat-topped pulse split equally into a positive and negative half. When there are no dynamics external to the tracker, this force program with their "muscle" produces the double-parabola response segment shape. While this simulates to some extent the physiological behavior of the force applied to the arm and the hand response found in compensatory tracking, it does not significantly affect the frequency response of the model since:

(i) A straight line drawn through the endpoints of a double parabola response differs only slightly from the double-parabola.

(ii) The dynamic control action of the intermittent model is unaffected by the particular shape of the output between sample points because the output is sampled only at the end of the execution of a program segment. Obviously, any output shape that coincides with the endpoints of the muscle response segments will lead to the same control action since the samplers will be totally unaware of it.

Therefore, for simplicity, the single integrator muscle and flat topped force program is used. Naturally the first derivative of the output under these conditions will be the train of flat-topped force program pulses and would differ from the derivate of the double parabola segment response which would be triangular pulses. However, this difference is so easily reconciled that it was not considered material.
} 


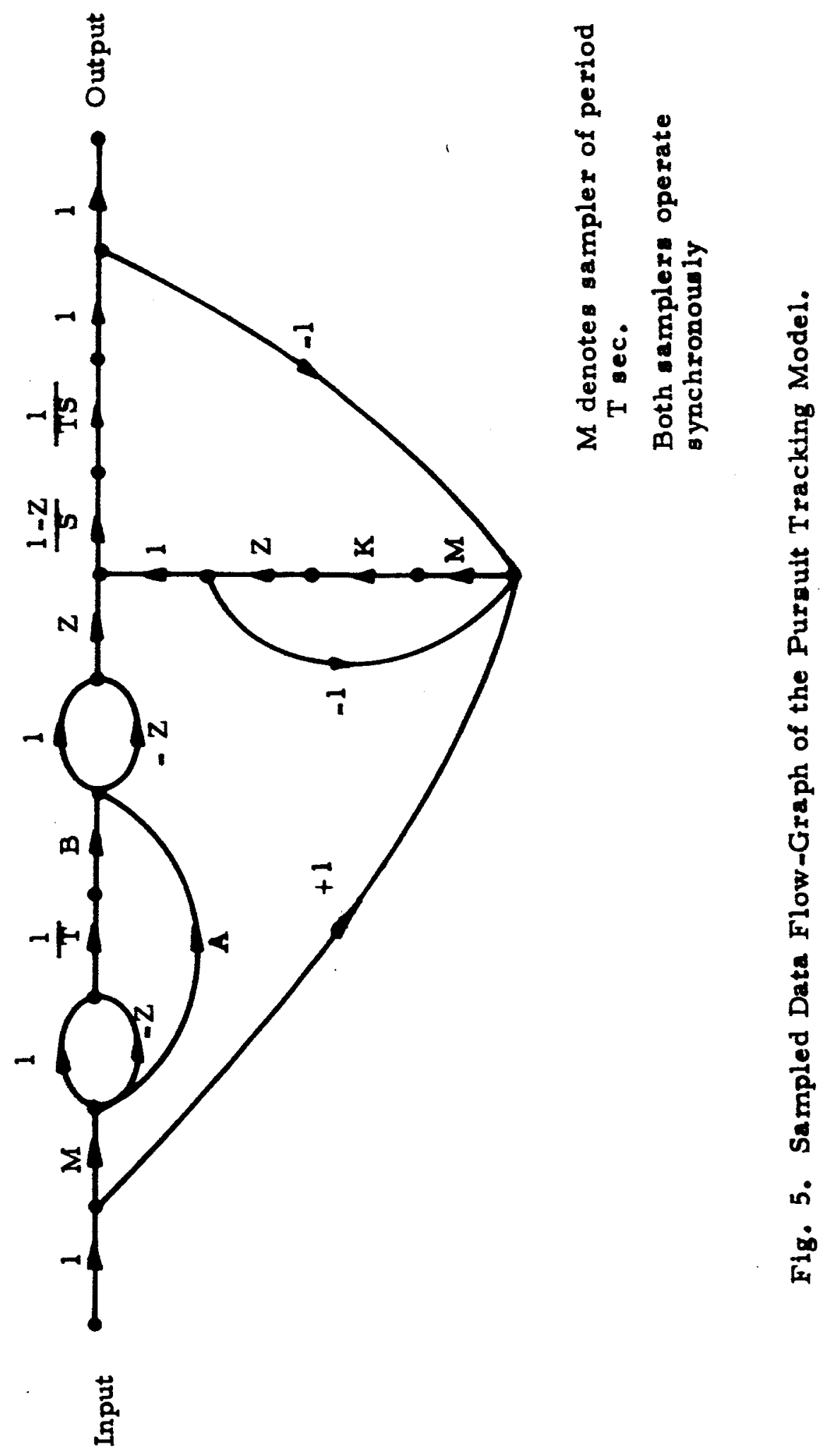


$+{ }^{+}$Elkind, Kelly and Payne propose a continuous differentiation to produce the exact Instantaneous error velocity for prediction in their compensatory hand tracking model. Arguing that velocity appears to be directly perceived. Westcott and Lemay also use a differentiation to produce instantaneous error velocity for prediction in their compensatory model. However, both Young and Bekey use a sample method for deriving the velocity estimate.

The discrete estimation of velocity sems more attractive for these reasons:

(i) The human can track as though it were completely continuous, an output from a sample-hold operation with a short sample period on a continuous ignal. Such an output consists of a "stalr-case" approximation to the continuous signal and for a sort ample period the approximation is excellent. A continuous differentiator operating on uch a stair-case input would produce nothing but spikes and extensive sophisticated filtering would be needed possibly prior to and following the differentiator in order to produce meaningful velocity information. However, a discrete velocity estimator is not affected by a staix case approximation and would give an a verage velocity estimate differing only slightly from its operation on the continuous signal.

(i1) It is well known that estimation of time-derivatives higher than velocity are very poor and probably not significantly useful in continuous tracking. A sampled derivative estimator requires memory in forming its estimate where the required memory space increases with the derivative order. For instance, a velocity estimator holds the past position sample in memory to compare with the present value. A discrete acceleration estimator would require either the preceding two input samples or the previous velocity estimate. Either way, increasing memory space is needed and the derivative approximations rapidly deteriorate and become unuaeful since they are compounded out of information necessarily extending farther into the past. Such considerations could plausibly Indicate why velocity seems to be the highest ignificant derivate used in tracking.

(iii) As the frequency range of the input is increased, the difcrete velocity estimation rapidly deteriorates for a fixed sampling rate ince the input changes significantly between ample periods with the possibilities of input reversals actually taking place between mamples. The continuous differentiator is, of course, not inherently less accurate at higher frequencies. It appears that velocity estimation in the human become poorer with increasing frequency. The discrete estimator method coincides nicely with this fact by its nature, whereas the continuous differentiator would require additional filtering to represent this. 
4. The operator acts directly on the error, to reduce it. This assumption was made to simplify the initial pursuit mode1.

5. The operator forms his programs from operations on the input and error synchronously every 0.2 second. This corres ponds to sampling the input and error synchronously at a rate of 5 samples per second. This frequency would correspond closely to the frequency of the segments observed in the pursuit tracking velocity record of Fig. 3.

Conceptual Operation of the Model

Figure 4 presents a block diagram of the model which embodies the elements specified above. The input is sampled every $T$ seconds. The samples representing instantaneous input position are passed to one circuit which weights them by a coefficient A. A parallel circuit subtracts the past value of the position and divides by the time between samples to give an approximation to the instantaneous velocity $(\Delta X / \Delta T)$. This velocity estimate is then weighted by a coefficient $B$. The sum of $A(X)$ and $B(\Delta X / \Delta T)$ represents the prediction done on the input. Because of the operation of the "muscle" which is a non-resetting integrator, just the differences in the prediction values must be sent to it. That is, if the prediction value does not change over a sample period, a zero value is passed to the summer through the 0.2 second delay.

The error is sampled and weighted and also passed to the summer through a 0.2 second delay. The delay is incorporated in each path separately since the internal stabilizing loop in the error path feeds back from the delayed error. The "memory feedback" loop represents an operator's ability to remember the error over one sample period so as to compensate for the effective delay of the muscle of one sample period.

The operation of the model is then as follows:

The input is sampled and the instantaneous position and estimated velocity computed and weighted separately. The changes in these quantities from the last sample instant are computed. The delay of 0.2 seconds represents this computation time and any neural reaction time. This computed information is sent to the muscle in the form of a command 
pulse of width 0.2 seconds and constant height. The muscle integrates this pulse to arrive at a new position. If any error results at the end of this movement, the error sampler takes this value and after one delay time adds it to the next command from the open-loop prediction path to the muscle for correction.

The memory feedback stabilizing loop is identical in purpose to that used by Lemay and Westcott in their step tracking compensatory model.

\section{Analytical Transmittance of the Model}

The block diagram in Fig. 4 can be translated into the sampled-data flow graph shown in Fig. 5. In this figure $M$ denotes the sampling operation and $\mathrm{Z}$ is defined as:

$$
Z=e^{-S T}
$$

where

$$
T=0.2 \mathrm{sec} .
$$

The three free parameters $A, B$ and $K$ represent respectively the weighting on position, velocity estimate and error. After suitable reduction by sampled-data techniques, the model transmittance can be found to be:

$$
\begin{aligned}
T_{R}=M\left[A+B\left(\frac{1-Z}{T}\right)\right]\left[\frac{1-Z}{S}\right]^{2} \frac{Z}{T}+ \\
\qquad \frac{\left[1-\left[A+B\left(\frac{1-Z}{T}\right)\right]\left[\frac{1-Z}{S}\right] \frac{Z}{T}\right]^{*} K Z\left(\frac{1-Z}{S}\right)\left(\frac{1}{S T}\right)}{1+[K Z]^{*}+\left[K Z\left(\frac{1-Z}{S}\right)\left(\frac{1}{S T}\right)\right]^{*}}
\end{aligned}
$$


where the starred brackets indicate that the sampled equivalents are to be found. For instance:

$$
\begin{gathered}
{[\mathrm{Kz}]^{*}=\mathrm{Kz}} \\
{\left[\mathrm{KZ}\left[\frac{1-\mathrm{Z}}{\mathrm{s}}\right]\left[\frac{1}{\mathrm{sT}}\right]\right]^{*}=\frac{\mathrm{Kz}^{2}}{1-\mathrm{Z}}}
\end{gathered}
$$

See Fig. 6 for a graphical derivation of Eq. (8)

$$
\left\{\left[A+B\left(\frac{1-Z}{T}\right)\right]\left[\frac{1-Z}{S}\right]^{2} \frac{Z}{T}\right\}^{*}=\left[A+B\left(\frac{1-Z}{T}\right)\right] z^{2}
$$

Therefore the Laplace transform of the output $[G(S)]$ can be expressed as

$$
G(S)=F(S) T_{R}
$$

or since

$$
\begin{gathered}
{[F(S)] M=[F(S)]^{*}=F(Z)} \\
G(S)=F(Z)\left\{\left[A+B\left(\frac{1-Z}{T}\right)\right]\left[\frac{1-Z}{S}\right]^{2} \frac{Z}{T}+\right. \\
\frac{\left[1-\left[A+B\left(\frac{1-Z}{T}\right)\right] Z^{2}\right] K Z\left(\frac{1-Z}{S}\right)\left(\frac{1}{S T}\right)}{1+K Z+\frac{K Z}{1-Z}}
\end{gathered}
$$

The imple denominator indicates a system stable for $\mathrm{K} \leq 2$. 


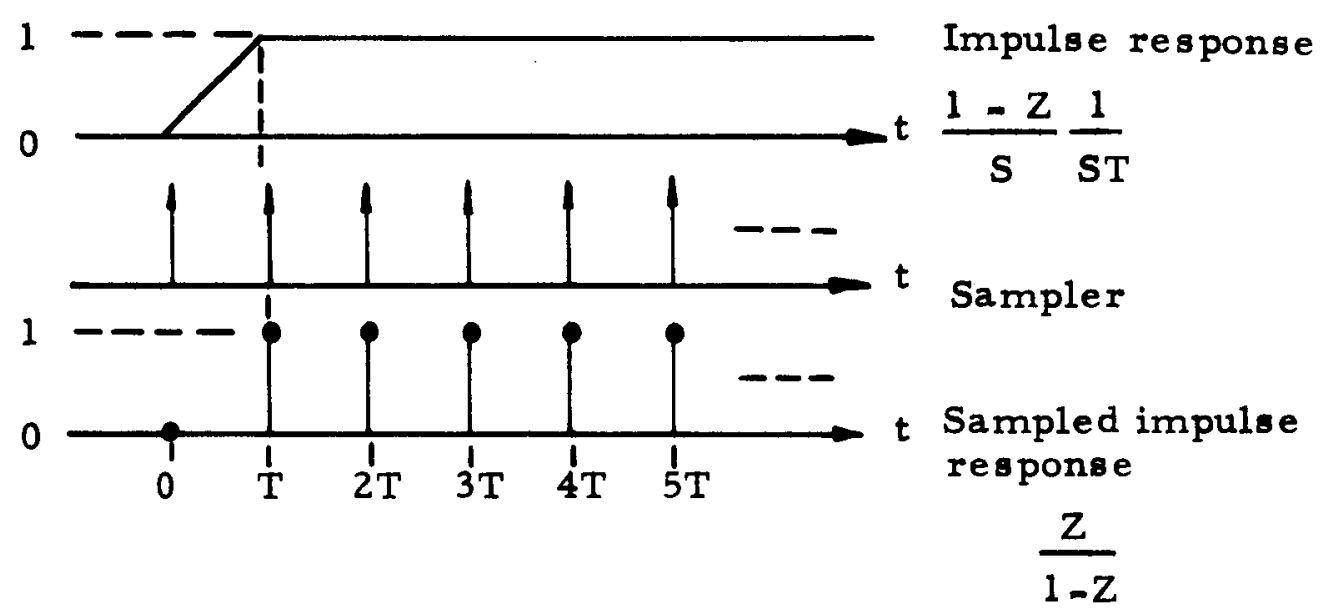

Fig. 6. Graphical Representation for Deriving the Sampled Equivalent of the Impuse Response $(1-\mathrm{z}) / \mathrm{ts}^{2}$. 
Experimental Evaluation of the Sampled Data Pursuit Model

The rather complex form of Eq. (12) badicates why an analytic derivation of the frequency cheracteristica was not pursued. Instead, a special purpose analog computer was bullt which had essentially the same transmittance as Eq. (6). The amplitude and phase characteristics of the model were then obtained through the use of an on line analyais using a digital computer in the M.I.T. Electronics Syatem. Laboratory.

The input power spectrum used to evaluate the model was flat to a sharp cutoff at $0.67 \mathrm{cps}$. This was used since Elkind's deta ${ }^{1}$ sowed nearly 100 per cent linear behavlor for humans tracking equal power epectra of approximately $0.64 \mathrm{cps}$ and below. As the model 1s lineax, this seemed 11ke Its most severe tent.

Results of Model Evaluation

1. Frequency Characteristica

Several transfer characterietics for the model using the $0.67 \mathrm{cps}$ input - pectrum are shown in Fig. 7 through 10. The effect of the three parametere can be observed in these figures. Figure 10 shows the closest agreement to Elkind's data. It should be remembered that Elkind's data reflect the averages for well-trained ubjects. Figure 10 also show the average of 4 run obtained from an untrained subject taken for comparison.

A. can be seen in Fig. 10, there is very good agreement in matching the particular shape of the humnn pursult tracking characteriatics. That 1s, the a mplitude characteriatic dips before rising at high frequency while the phase characteristic flattens out. The flattening characteristic is mainly due to velocity coefficlent $B$.

\section{Time Response Characteriftice}

For further comparison, a trained subject tracked in pursuit fashion an input composed of five sinusoids from 0.05 to 0.6 cps while the analog model tracked the same input in parallel. The recorda of input, human response and model response are shown in Fig. 11. The close agreement In human and model response can be seen particularly when the input approaches a local velocity minimum but does not reverse direction. At such points, the human expects a reversal and beglns or actually does execute one. This same behavior is also displayed by the model. Both, then, perform imilar over anticipative responses. Several such res. ponses are marked in Fig. 11. 


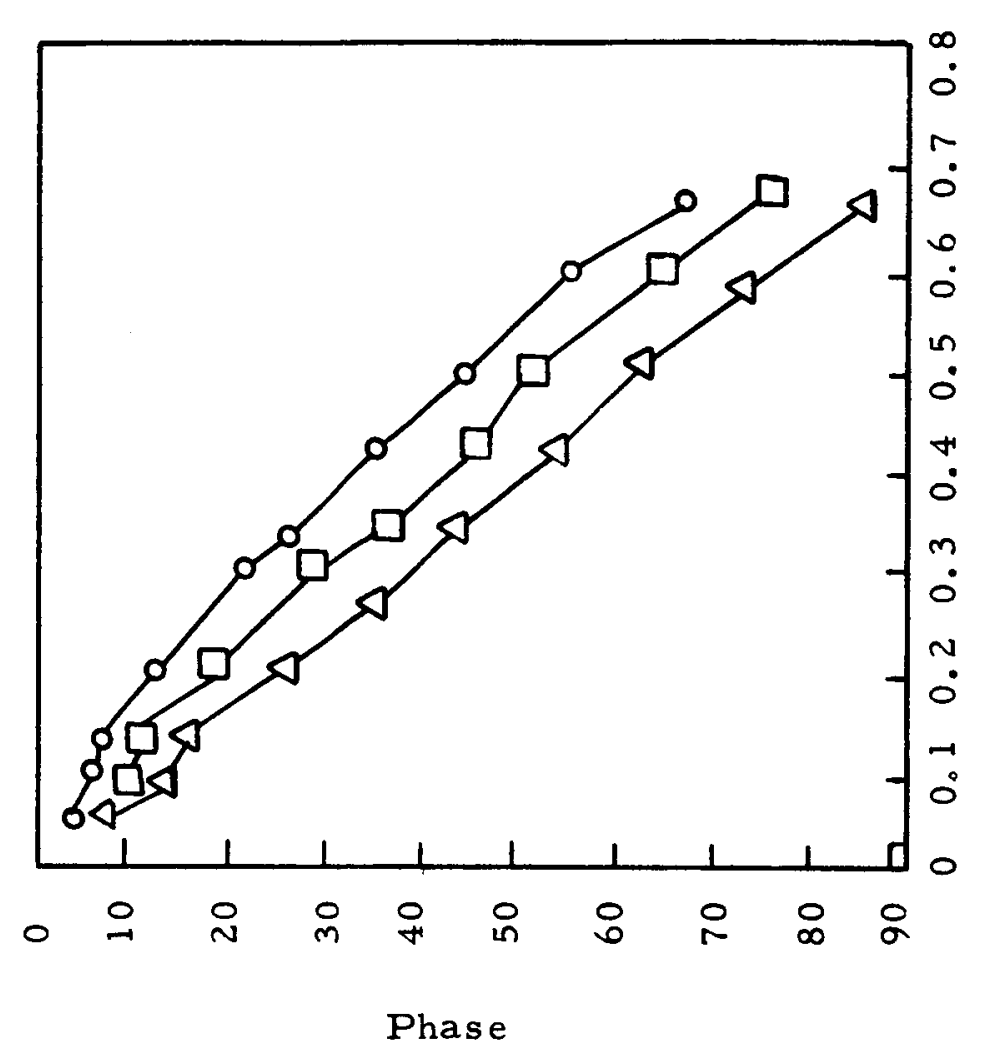

$$
\begin{aligned}
& 000 \\
& \text { ต田品 } \\
& \text { in } N \\
& \text { ○ } 0 \\
& \text { " } 4 \text { " } 4
\end{aligned}
$$

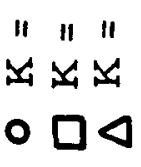$$
\text { un }
$$

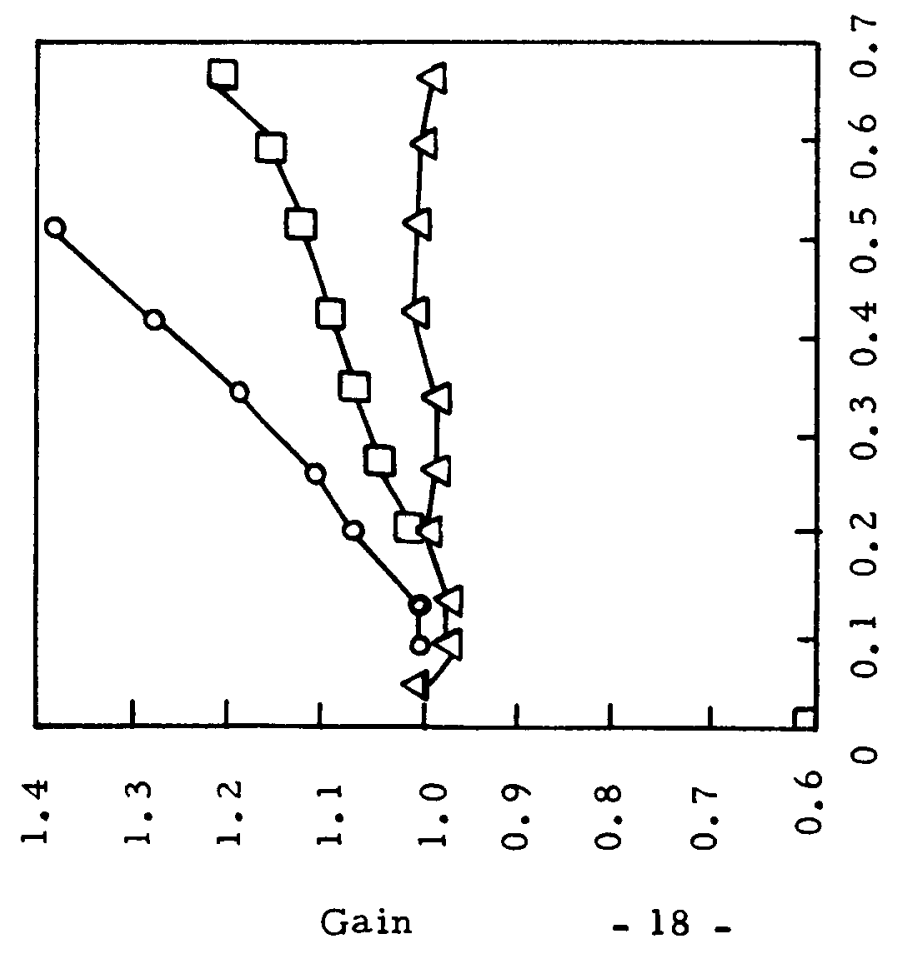

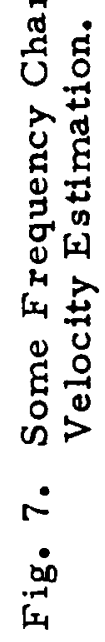




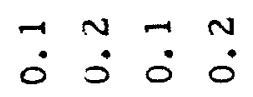

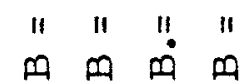

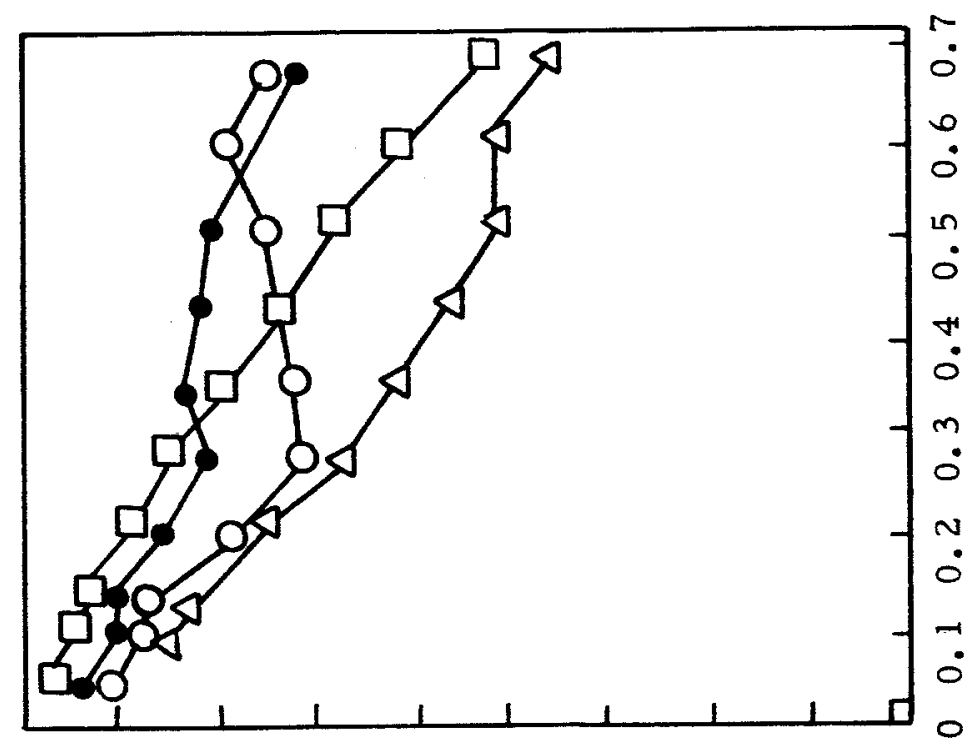

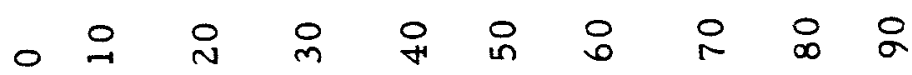

Phase
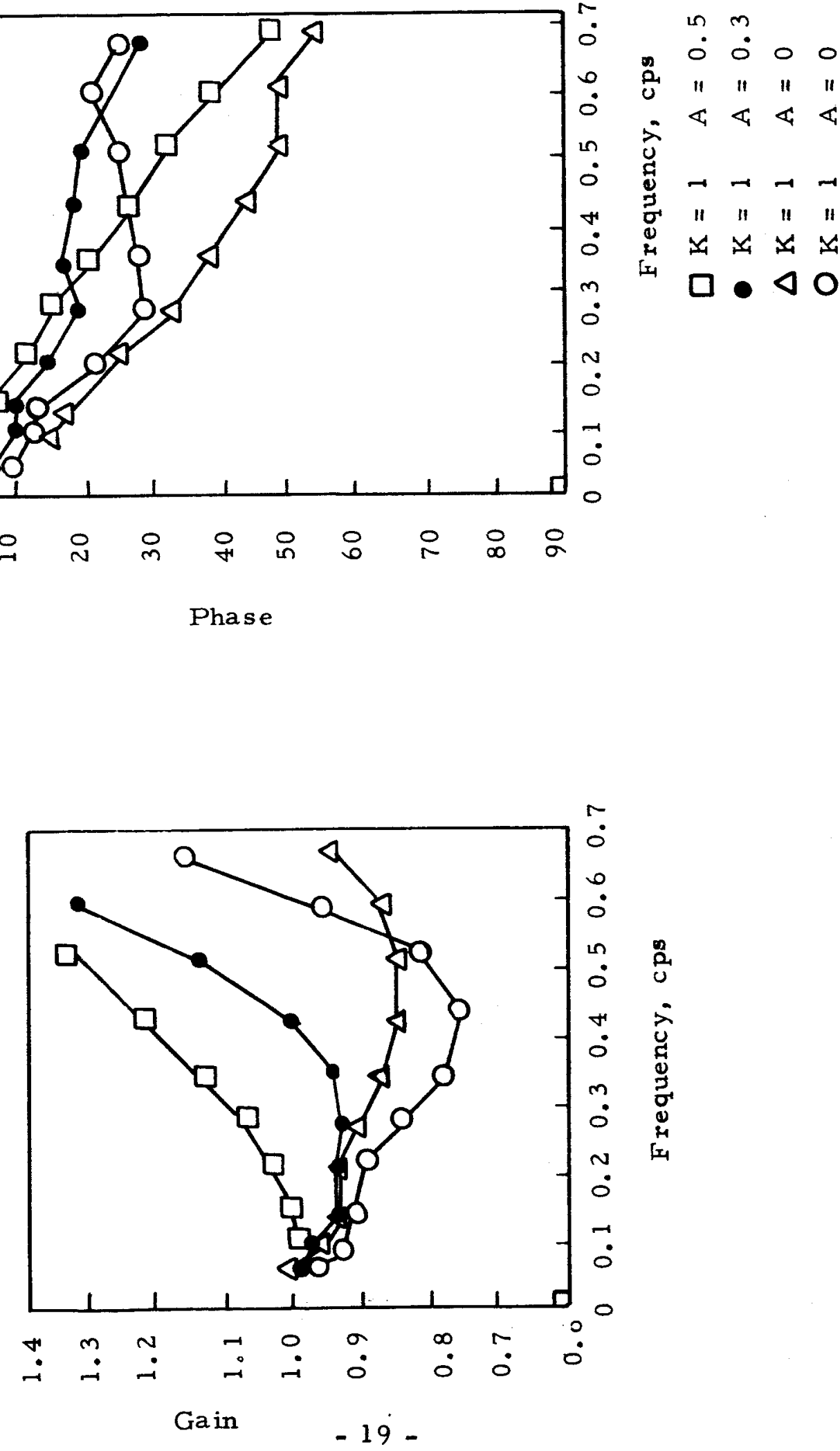


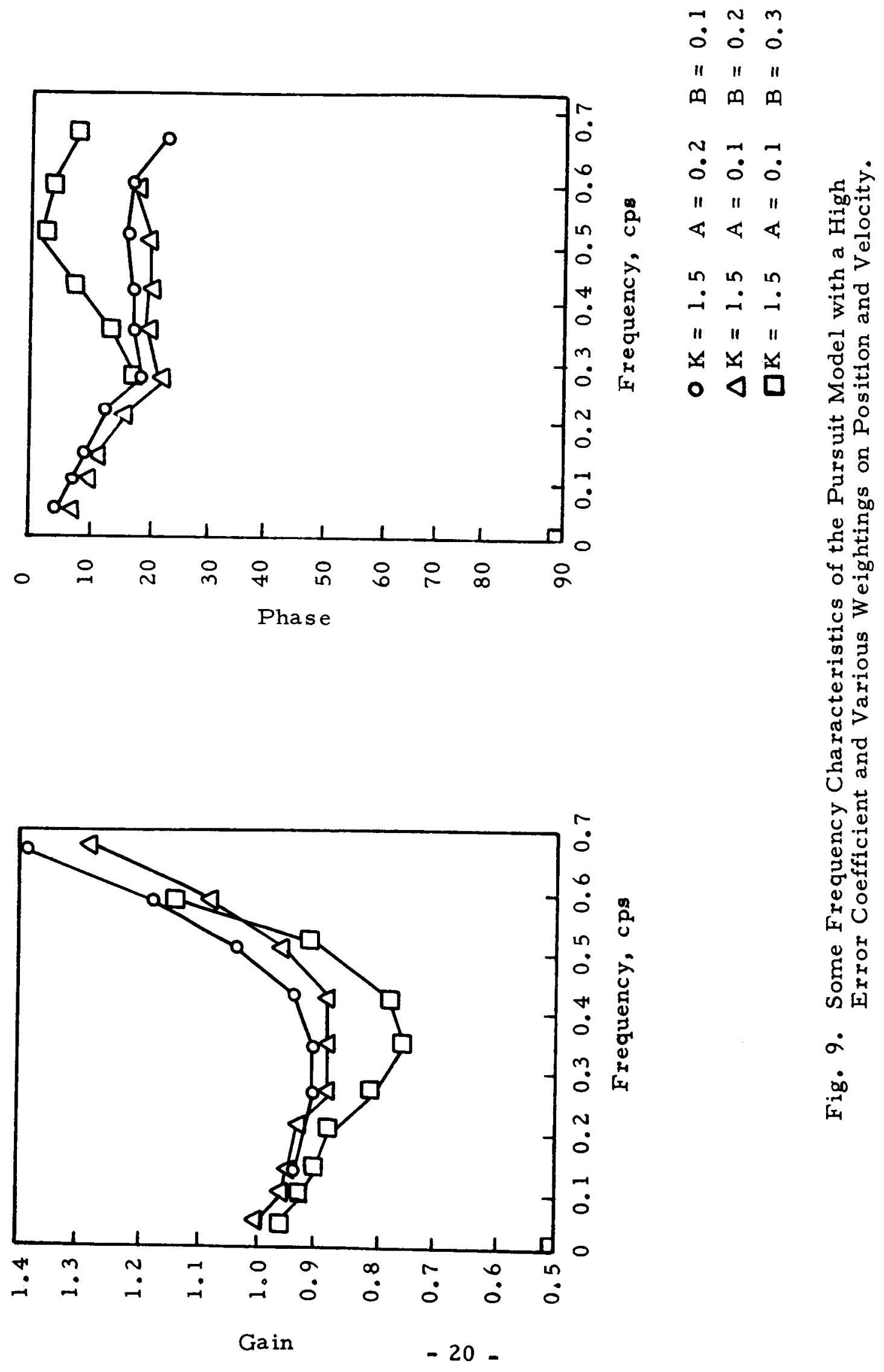




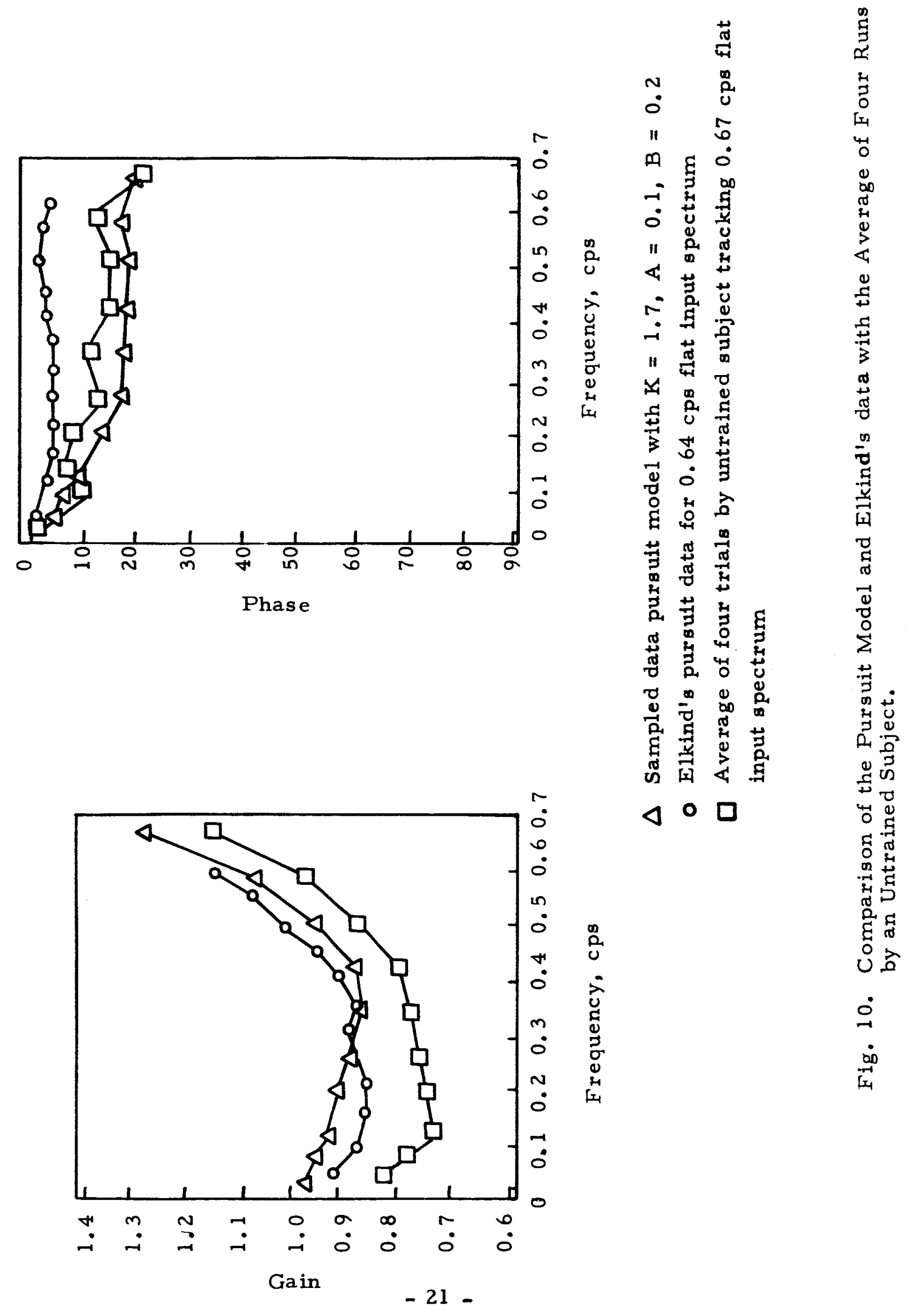




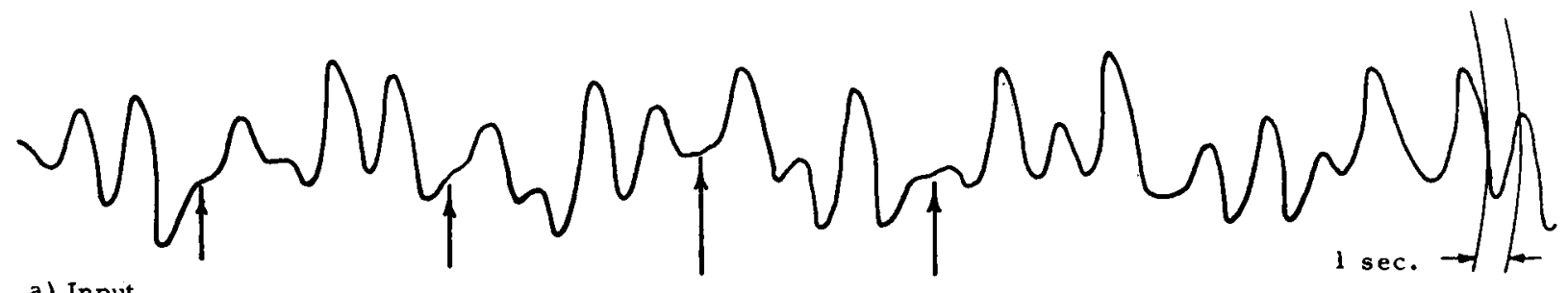

a) Input

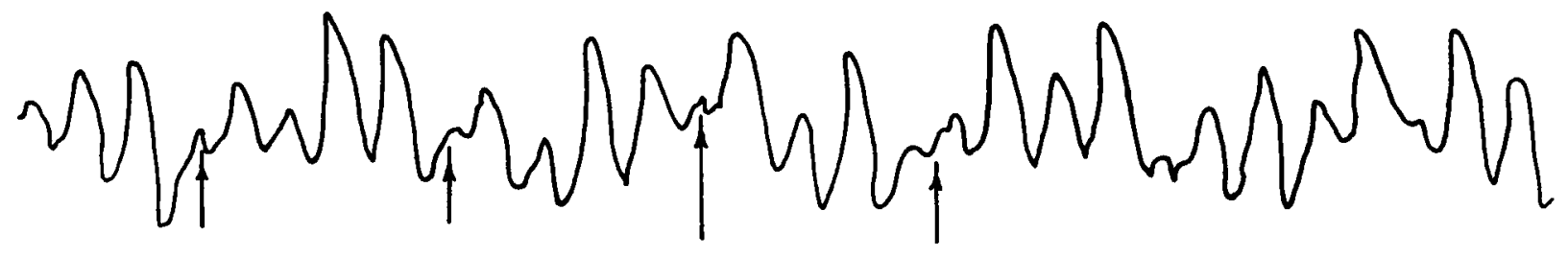

b) Model

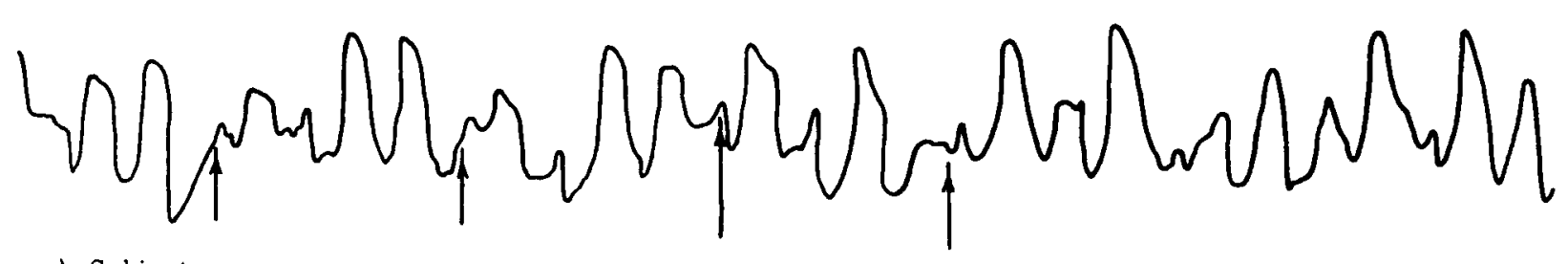

c) Subject

Fig. 11. Comparison of Time Responses of a Human Pursuit Tracker and the Model Following the Same Input. Arrows indicate characteristic reversals of human response which pursuit sampled data model mimics but which linear model smoothes. 


\section{Conclusions}

The model appears a good representation of human pursuit tracking of random continuous signals limited to approximately $0.7 \mathrm{cps}$ and below, based upon the simllarity of time domain responses and the general duplication of the shape of human amplitude and phase pursuit characteristics. The amplitude characteristic of empirtcal data was closely matched, although the model had about a 15 degree greater phase lag than a well-trained human. Further adjustment can obviously be performed to bring the model into closer agreement with human data. For instance, some more elaborate operation on the error could be added. 
1. Elkind, J.I., "Characterlstics of Simple Manusl Control Systems," Lincoln Laboratory Report No. 111, M.I. T. Laboratory, Lexington, Massachusetts, April 1956.

2. Elkind, J.I., Kelly, J.A., and Payne, R.A., "Adaptive Characteristics of the Human Controller in Systems Having Complex Dynamics," Fifth National Symposium on Human Factors in Electronlcs, PTG, HFE, IEEE, San Diego, California, May 5 - 6, 1964.

3. Young, L.R., "A Sampled-Data Model for Eye Movements," Thesis (ScD), Aeronautic Engineering Department, M.I.T., Cambridge, Massachusetts, June 1962.

4. Bekey, G.A., "Sampled-Data Models of the Human Operator in a Control System", Space Technology Laboratories, Inc., January 15, 1962.

5. Lemay, L.P., and Westcott, J.H., "The Simulation of Human Operator Tracking Using an Intermittent Model, "International Congress on Human Factors in Electronics, IRE PGHT, May 1962. 